\title{
Peter SAHLINS, Unnaturally French. Foreign Citizens in the Old Regime and after
}

\section{Marc Belissa}

\section{OpenEdition \\ Journals}

Édition électronique

URL : https://journals.openedition.org/ahrf/10322

DOI : 10.4000/ahrf.10322

ISSN : $1952-403 X$

Éditeur :

Armand Colin, Société des études robespierristes

Édition imprimée

Date de publication : 15 mars 2006

Pagination : 235-236

ISSN : 0003-4436

Référence électronique

Marc Belissa, "Peter sahıINs, Unnaturally French. Foreign Citizens in the Old Regime and after », Annales historiques de la Révolution française [En ligne], 343 I janvier-mars 2006, mis en ligne le 17 novembre 2008, consulté le 23 avril 2022. URL : http://journals.openedition.org/ahrf/10322 ; DOI https://doi.org/10.4000/ahrf.10322

Ce document a été généré automatiquement le 23 avril 2022

Tous droits réservés 


\title{
Peter SAHLINS, Unnaturally French. Foreign Citizens in the Old Regime and after
}

\author{
Marc Belissa
}

\section{RÉFÉRENCE}

Peter Sahlins, Unnaturally French. Foreign Citizens in the Old Regime and after, Ithaca \& London, Cornell University Press, 2004, XIV - 454 p., ISBN 0-8014-4142-0, 29.95 \$.

1 Le présent ouvrage s'inscrit dans la suite des problématiques développées dans un précédent livre (au titre calamiteux de l'aveu même de Peter Sahlins!) Et si on faisait payer les étrangers? Louis XIV, les immigrés et quelques autres écrit avec Jean-François Dubost. Il s'agit pour l'auteur de faire l'histoire des théories, des procédures, des pratiques et des représentations de la naturalisation dans la France de l'Ancien Régime jusqu'à ce qu'il appelle la période de la « révolution de la citoyenneté » qui commence grosso modo au milieu du XVIII ${ }^{e}$ siècle pour s'achever en 1819, date symbolique qui marque l'abolition définitive du droit d'aubaine dans la France contemporaine. Le projet se veut à la confluence de l'histoire juridique, politique et sociale. En effet, l'auteur s'intéresse tout d'abord à la construction par les juristes d'un modèle « absolutiste » de la citoyenneté (absolutist model of French citizenship) aux XVI ${ }^{\mathrm{e}}$ et XVII ${ }^{\mathrm{e}}$ siècles, modèle soumis à la critique des Lumières au XVIII ${ }^{\mathrm{e}}$ siècle, puis détruit par la Révolution et réutilisé en partie par Napoléon et Louis XVIII. Les sources utilisées permettent également de dresser un portrait social de ces étrangers en mal de naturalité française. Leur origine géographique, leurs identités changeantes dans le temps et dans l'espace du royaume de France, forment la matière de la deuxième partie de l'ouvrage. Enfin Peter Sahlins s'intéresse à la politique de naturalisation menée par le Conseil du roi, ses principaux ministres et officiers, ainsi qu'aux processus concrets de la naturalisation. Bien que ces procédures ne concernent qu'un nombre restreint d'individus dans la période considérée (tous ceux qui ne demandent pas leur 
naturalisation restent en dehors des sources), leur histoire est un observatoire de premier ordre pour comprendre celle, plus globale, de l'émergence de la "nationalité » et des débuts de l'immigration contemporaine.

Peter Sahlins avait déjà utilisé une partie des sources mobilisées ici (il s'agit tout d'abord des rôles de la taxe imposée par Louis XIV aux étrangers entre 1697 et 1707, puis d'un corpus de plus de 6000 lettres de naturalité entre 1660 et 1790) dans un article publié dans les Annales HSS en 2000 et repris dans ce nouvel ouvrage. Il y a ajouté un important corpus juridique théorique autour de la notion de citoyenneté sous l'Ancien Régime. En effet, cette histoire de la naturalisation s'insère dans une perspective large qui est celle du passage de la catégorie juridique du "citoyen » d'Ancien Régime (définie par opposition à celle de l'étranger) à la citoyenneté "politique » issue des Lumières et de la Révolution française. Dans cette histoire, un élément juridique joue un rôle extrêmement important : c'est le «droit d'aubaine », droit féodal mineur à l'origine, qui revient à tous moments comme mécanisme fondamental de la construction par la monarchie absolue d'une distinction entre citoyens et étrangers.

3 Sous l'Ancien Régime, le droit ne définit pas le «citoyen » comme un sujet possédant des droits civils ou politiques mais comme un individu "n'étant pas limité » par les "incapacités légales» touchant les étrangers (ce que l'auteur appelle les "antiprivilèges »). Les étrangers ne pouvaient en effet tenir des offices ou des bénéfices ecclésiastiques et pouvaient être soumis à des taxes spécifiques ou à des incapacités, partielles ou totales, dans le domaine économique; mais la plus gênante des contraintes, pour les possédants, était le «droit d'aubaine» qui limitait leur pouvoir d'hériter ou de transmettre des biens. Peter Sahlins montre de manière extrêmement convaincante comment ce droit a été utilisé par la monarchie aux XVI ${ }^{e}$ et XVII ${ }^{e}$ siècles pour construire et étendre la catégorie juridique de Français «non-naturel » (d'où le titre de l'ouvrage) et par conséquent pour «créer » symétriquement une citoyenneté « absolutiste » pour les sujets « naturels » du roi. C'est donc à l'aide d'un droit féodal étendu largement au-delà de ses origines que la monarchie a construit un droit de la naturalité. Ce processus est, selon l'auteur, typique de l'histoire de la France, puisque dans la plupart des États européens à la même époque, ce sont les échelons locaux qui élaborent de manière plus ou moins « silencieuse » une jurisprudence de la naturalité.

4 La lettre de naturalité, étudiée de manière exhaustive par Peter Sahlins dans sa structure comme dans sa rationalité juridique, connaît un premier moment d'élaboration vers 1570 au moment où le modèle bodinien du citoyen prend forme et un second moment pendant le règne de Louis XIV, culminant dans la fameuse taxe de naturalisation de 1697, déjà étudiée par l'auteur dans son ouvrage précédent. De 1697 à 1750 , le droit d'aubaine et la naturalisation fonctionnent comme des procédures légales et administratives relativement routinières. L'étranger naturalisé «ne cesse pas » d'être un étranger, il devient seulement un «citoyen étranger», car la naturalisation est conçue comme une "fiction juridique» qui ne modifie pas "réellement» la "nature» de l'individu. Dans ce processus, le "patriotisme» ou la profondeur du sentiment « d'assimilation » à la « nation » française ne joue strictement aucun rôle, bien que les motivations affichées des étrangers désirant être naturalisés nous donnent quelques éléments sur leur statut (plutôt que sur leur "intégration ", terme que Peter Sahlins considère, à juste titre, comme anachronique). 
5 Le modèle «absolutiste » de la naturalité est soumis à la critique à partir des années 1750 ; c'est le début de la « révolution de la citoyenneté » selon Peter Sahlins. À partir du tournant des Lumières et surtout de la guerre de Sept Ans, les débats autour de la citoyenneté passent du terrain du droit à celui de la politique avec l'irruption bien connue de la figure du citoyen dans les débats sur les Parlements et dans les querelles religieuses. Peter Sahlins montre parfaitement la concomitance de l'émergence de la citoyenneté des Lumières avec l'abolition systématique du droit d'aubaine réalisée par le Ministère des Affaires étrangères dans une série impressionnante de traités et de conventions spécifiques dont la liste figure en annexe de l'ouvrage. Ces deux phénomènes sont par ailleurs mis en relation avec le travail de délimitation de l'espace de la souveraineté réalisé par les commissions de limites de la monarchie, visant à instituer la frontière comme ligne de séparation entre les "naturels» et les " étrangers » et non plus comme une zone de confins.

6 Droit fustigé par les philosophes et les économistes comme un reste de la barbarie féodale, le droit d'aubaine n'est néanmoins pas abrogé universellement par la monarchie qui entend rester sur le terrain de l'abolition bilatérale avec les États qui demandent ce privilège pour leurs naturels. En abolissant, même de manière incomplète, le droit d'aubaine, la monarchie contribue, sans le vouloir, à l'émergence du modèle révolutionnaire universel de la citoyenneté en délégitimant cet élément fondamental de la définition du « citoyen absolutiste ». En effet, la Révolution abroge le droit d'aubaine, et ceci à quatre reprises entre 1790 et 1791 (en tant que droit féodal, en tant que privilège, en tant que prérogative souveraine et enfin dans les colonies). Au nom d'une conception cosmopolitique de la citoyenneté et de l'intérêt du commerce entre les hommes, Barère demande à l'Assemblée constituante d'en finir avec ce « droit barbare ».

7 Comme en bien d'autres cas, Bonaparte opère en 1803 un large retour en arrière en réinstituant un droit d'aubaine (mais beaucoup plus limité que sous l'Ancien Régime) soumis à un régime d'exemptions diplomatiques bilatérales au nom de l'intérêt national étroit. Cette réinterprétation du droit d'aubaine est une fois de plus concomitante avec une redéfinition de la citoyenneté: c'est l'invention de la «nationalité » dans le Code civil, sujet déjà abordé par de nombreux spécialistes et notamment par Patrick Weil dans Qu'est-ce qu'un Français?, mais que Peter Sahlins replace de manière extrêmement convaincante dans un cadre chronologique plus large. Il revient à la Restauration d'abolir définitivement le droit d'aubaine en 1819 à l'issue d'un débat qui reprend en partie les éléments précédents.

8 L'ouvrage de Peter Sahlins montre parfaitement que le débat autour de la naturalité ne se résume pas à une opposition largement anachronique entre droit du sol et droit du sang. Pour l'Ancien Régime, cette dichotomie n'est pas opératoire et ne permet pas de comprendre la teneur de la construction d'une citoyenneté « absolutiste » qui se situe sur un autre plan que celui du droit individuel.

9 Du côté des critiques, le spécialiste de la Révolution française pourra trouver que l'auteur n'intègre pas assez la dimension cosmopolitique de la question de la citoyenneté des étrangers entre 1789 et 1800 . Ce qui est assez paradoxal pour un historien qui traite brillamment des rapports entre les Français et les peuples voisins par le biais de la naturalisation. Pour ne citer qu'un exemple, l'auteur ne parle pas des projets d'intercitoyenneté entre les "républiques libres" proposés par nombre de révolutionnaires, qui avec l'Angleterre, qui avec les États-Unis entre 1789 et 1795, puis 
sous le Directoire, avec les Républiques-sœurs. En effet, Peter Sahlins se contente en la matière de reprendre une forme de récit-type du passage du cosmopolitisme des années 1789-1792 à une conception plus restrictive de l'accès des étrangers à la citoyenneté française pendant la République montagnarde. On pourra également discuter quelques affirmations, sans doute reprises des travaux de Vida Azimi en particulier, sur la définition du citoyen dans la Constitution de 1793, qui, selon l'auteur, anticiperait la définition de la «nationalité » de 1803. Pour ma part, j'en suis très peu convaincu. De même, si Peter Sahlins évoque assez rapidement le cas de plusieurs étrangers naturalisés émettant des réclamations contre la loi du 26 germinal an II, on ne peut en déduire, comme semble le faire l'auteur, qu'il s'agit là de cas qui prouveraient la fermeture de la naturalisation pendant la période de la Convention montagnarde puisque, au dire même de l'auteur, les réponses des autorités à ces réclamations sont inconnues.

10 Malgré ces quelques remarques critiques, l'ouvrage de Peter Sahlins est sans aucun doute un livre important. Il renouvelle considérablement le débat autour de l'émergence de la nationalité et permet de considérer les débats révolutionnaires dans une perspective chronologique plus large et surtout plus volontiers tournée vers l'époque moderne. 